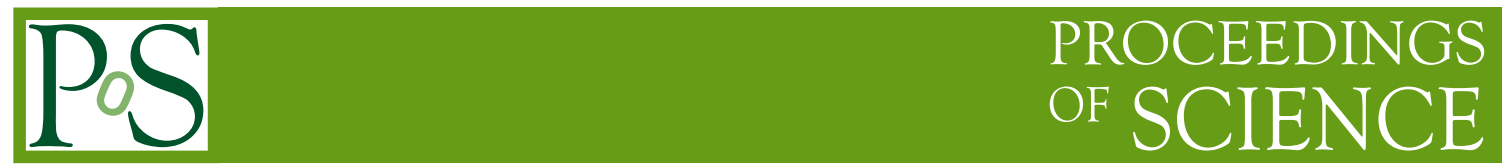

\title{
Charged Higgs effects on top spin correlations
}

\author{
David Eriksson* \\ Uppsala University \\ E-mail: david.eriksson@physics.uu.se
}

Discovery of a charged Higgs requires that its spin and couplings are determined. We analyze how charged Higgs effects on spin correlations in top quark pair production can be used for determination of the helicity structure of the Yukawa couplings. For a general Two Higgs Doublet Model, we determine spin analyzing coefficients for top decays involving charged Higgs and study in detail the effect on the top spin correlations. The influence for a charged Higgs is largest in the high and low tan beta limits, where differences from the SM are most significant.

Prospects for Charged Higgs Discovery at Colliders

16-19 September 2008

Uppsala, Sweden

* Speaker. 


\section{Introduction}

To claim the discovery of a charged Higgs boson one needs to measure its spin. We have here presented one possible way to detect the coupling structure of the charged Higgs Yukawa couplings based on the work [1]. For more details and references see [1].

\section{Top spin correlations}

At hadron colliders top quarks are mostly produced in pairs. There are two production modes, quark induced and gluon induced. So far top quarks has only been produced at the Tevatron and there quark induced production is dominant. When the LHC starts to produce top quark pairs the dominant mode will be gluon induced.

Top quarks are very short lived and decays before any top hadrons are formed. In a pair production the spin of the two top quarks are correlated and this spin information will propagate to the decay products since no intermediate top hadron was produced.

A parton level correlation can be defined [2] as

$$
\hat{C}_{i j}\left(M_{t \bar{t}}^{2}\right)=\frac{\hat{\sigma}_{i j}\left(t_{\uparrow} \bar{t}_{\uparrow}+t_{\downarrow} \bar{t}_{\downarrow}\right)-\hat{\sigma}_{i j}\left(t_{\downarrow} \bar{t}_{\uparrow}+t_{\uparrow} \bar{t}_{\downarrow}\right)}{\hat{\sigma}_{i j}\left(t_{\uparrow} \bar{t}_{\uparrow}+t_{\downarrow} \bar{t}_{\downarrow}\right)+\hat{\sigma}_{i j}\left(t_{\downarrow} \bar{\tau}_{\uparrow}+t_{\uparrow} \bar{t}_{\downarrow}\right)}
$$

where $t_{\uparrow}$ and $t_{\downarrow}$ indicates the two different polarizations a top quark can have. In this work we are working in the helicity basis where the spin of the quarks are quantized along the momentum of the top quark in the $t \bar{t}$ rest frame. At threshold we have $\hat{C}_{q \bar{q}}\left(4 m_{t}^{2}\right)=-\frac{1}{3}$ and $\hat{C}_{g g}\left(4 m_{t}^{2}\right)=1$. In the ultra-relativistic limit both $\hat{C}_{q \bar{q}}$ and $\hat{C}_{g g}$ goes to -1 .

\section{Charged Higgs in top decays}

In the SM the top quark decays almost exclusively to $W^{+} b$. If one introduces a charged Higgs the top can also decay to $H^{+} b$ if the charged Higgs is lighter than the top. The general form of the Lagrangian for charged Higgs interactions with fermions is

$$
\begin{aligned}
\mathscr{L}_{H}= & \frac{g_{W}}{2 \sqrt{2} m_{W}} \sum_{\{u, c, t\}} V_{u d}\left\{H^{+} \bar{u}\left[A\left(1-\gamma_{5}\right)+B\left(1+\gamma_{5}\right)\right] d+H^{-} \bar{d}\left[B^{*}\left(1-\gamma_{5}\right)+A^{*}\left(1+\gamma_{5}\right)\right] u\right\} \\
& +\frac{g_{W}}{2 \sqrt{2} m_{W}} \sum_{\{e, s, \tau\}}\left[H^{+} C \bar{v}_{l}\left(1+\gamma_{5}\right) l+H^{-} C^{*} \bar{l}\left(1-\gamma_{5}\right) v_{l}\right],
\end{aligned}
$$

where $A, B$ and $C$ are free parameters.

One way of introducing a charged Higgs is to extend the Standard Model Higgs sector by introducing a second Higgs doublet. These, so called, Two Higgs Doublet Models adds four new degrees of freedom which are manifest as four additional Higgs bosons, two neutral $H$ and $A$ and two charged $H^{ \pm}$. In the neutral sector a Lagrangian, similar to the one above, exists which in its most general form includes flavor changing neutral currents. To avoid this a discrete symmetry is imposed so that the different fermions only couple to one of the doublets. This can be done in different ways and the two mostly used are call type I, where one doublet couples to all fermions, 


\begin{tabular}{ccc}
\hline Coupling & $2 \mathrm{HDM}(\mathrm{I})$ & $2 \mathrm{HDM}(\mathrm{II})$ \\
\hline$A$ & $m_{u} \cot \beta$ & $m_{u} \cot \beta$ \\
$B$ & $-m_{d} \cot \beta$ & $m_{d} \tan \beta$ \\
$C$ & $m_{l} \cot \beta$ & $m_{l} \tan \beta$ \\
\hline
\end{tabular}

Table 1: Charged Higgs couplings to fermions in 2HDM type I and II.

\begin{tabular}{ccc}
\hline $\begin{array}{c}\text { Analyzing } \\
\text { particle }\end{array}$ & $W^{+}\left(\omega=m_{W}^{2} / m_{t}^{2}\right)$ & $H^{+}\left(\xi=m_{H^{+}}^{2} / m_{t}^{2}\right)$ \\
\hline$b$ & $-\frac{1-2 \omega}{1+2 \omega}$ & $-\frac{A^{2}-B^{2}}{A^{2}+B^{2}} f(\xi, A, B)$ \\
$W^{+} / H^{+}$ & $\frac{1-2 \omega}{1+2 \omega}$ & $\frac{A^{2}-B^{2}}{A^{2}+B^{2}} f(\xi, A, B)$ \\
$l^{+}(\bar{d})$ & 1 & $\frac{1-\xi^{2}+2 \xi \ln \xi}{(1-\xi)^{2}} \frac{A^{2}-B^{2}}{A^{2}+B^{2}} f(\xi, A, B)$ \\
$v_{l}(u)$ & $\frac{(1-\omega)\left(1-11 \omega-2 \omega^{2}\right)-12 \omega^{2} \ln \omega}{(1-\omega)^{2}(1+2 \omega)}$ & $-\frac{1-\xi^{2}+2 \xi \ln \xi}{(1-\xi)^{2}} \frac{A^{2}-B^{2}}{A^{2}+B^{2}} f(\xi, A, B)$ \\
\hline
\end{tabular}

Table 2: Spin analyzing coefficients for various decay products in decays of fully polarized top quarks, $\left(t_{\uparrow} \rightarrow b W^{+} / H^{+} \rightarrow b l^{+} v_{l}\right) . A$ and $B$ are part of the charge Higgs couplings and $f(\xi, A, B)$ is a threshold factor.

and type II, where up type fermions couple to one doublet and down types to the other. In table 1 the couplings $A, B$ and $C$ are given for type I and II. $\tan \beta$ is defined as the ratio between the vacuum expectation value for the two doublets. In the following we will only use the Two Higgs Doublet Model with type II couplings, 2HDM(II).

\section{Measurement of spin and correlations}

For the decay of a fully polarized top, the angular distributions of the decay products are related to the spin of the decaying top. It is possible to write the partial width $\Gamma\left(t_{\uparrow} \rightarrow b X^{+} \rightarrow b l^{+} v_{l}\right)$ as

$$
\frac{1}{\Gamma} \frac{\mathrm{d} \Gamma}{\mathrm{d} \cos \theta_{i}}=\frac{1+\alpha_{i} \cos \theta_{i}}{2}
$$

where $\alpha_{i}$ is the spin analyzing coefficient of decay product $i$. Table 2 shows these coefficients for the decay mediated by a $W^{+}$or a $H^{+}$. Two things can be noted about the charged Higgs coefficients. First that all are proportional to the couplings as $\frac{A^{2}-B^{2}}{A^{2}+B^{2}}$, which in the high (small) $\tan \beta$ limit goes to $1(-1)$. Secondly, there is a common threshold factor, $f(\xi, A, B)$, which is 1 except when $\xi=m_{H^{+}}^{2} / m_{t}^{2}$ is very close to 1 .

In $2 \mathrm{HDM}(\mathrm{II})$ the coefficients depend on $m_{H^{+}}$and $\tan \beta$ and in figure 1 this dependence is shown. The left and middle plot shows the dependence on the mass for two different $\tan \beta$ and the 

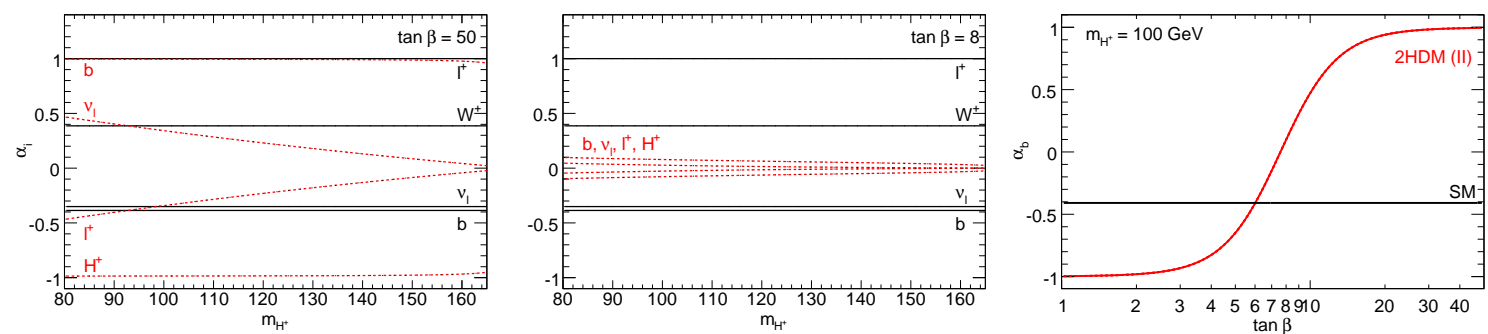

Figure 1: Spin analyzing coefficients $\alpha_{i}$ for 2HDM(II) in red and SM in black. The left plots shows the dependence on $m_{H^{+}}$for $\tan \beta=50$, the middle plots shows the dependence on $m_{H^{+}}$for $\tan \beta=8$ and the right plots shows the dependence of $\alpha_{b}$ on $\tan \beta$ for $m_{H^{+}}=100 \mathrm{GeV}$.

effect from the threshold factor is visible for large masses. The dependence on $\tan \beta$ is in the right plot where the behavior for large and small $\tan \beta$ is clearly visible.

From the parton level correlation 2.1 a correlation coefficient, $\mathscr{C}$, can be calculated by convoluting the expression with parton density functions and integration over the relevant mass spectrum of the pair. This coefficient enters the two dimensional correlation that can be defined as

$$
\frac{1}{N} \frac{\mathrm{d}^{2} N}{\mathrm{~d} \cos \theta_{i} \mathrm{~d} \cos \theta_{j}}=\frac{1}{4}\left(1+\mathscr{C} \alpha_{i} \alpha_{j} \cos \theta_{i} \cos \theta_{j}\right)
$$

where $\theta_{i}$ is the angle in the top rest frame between the decay product and the top momentum in the top pair rest frame. For the LHC the LO value for the coefficient is $\mathscr{C}=0.319$. A NLO calculation of this has been done [3] and the result is $\mathscr{C}=0.326$.

The two dimensional distribution is sensitive to phase space cuts. A quantity that is more robust against phase space cuts is the one dimensional correlation defined as

$$
\frac{1}{N} \frac{\mathrm{d} N}{\mathrm{~d} \cos \theta_{i j}}=\frac{1}{2}\left(1+\mathscr{D} \alpha_{i} \alpha_{j} \cos \theta_{i j}\right)
$$

where $\cos \theta_{i J}=\hat{p}_{i} \cdot \hat{p}_{j}$ and $\hat{p}_{i}$ are the unit vectors corresponding to the angles, $\theta_{i}$ above. At LO $\mathscr{D}=-0.216$ and the NLO value is $\mathscr{D}=-0.219[3]$.

The two correlations given above both requires the reconstruction of the complete event. In the case where the final state includes neutrinos this can be difficult to do. In [1] we instead introduced correlations which involve azimuthal angles which can be determined when only the transverse rest frame is reconstructed. A one dimensional correlation using azimuthal angles can be defined as

$$
\frac{1}{N} \frac{\mathrm{d} N}{\mathrm{~d} \cos \left(\Delta \phi_{i}-\Delta \phi_{j}\right)}=\frac{1}{2}\left[1+\mathscr{D}^{\prime} \alpha_{i} \alpha_{j} \cos \left(\Delta \phi_{i}-\Delta \phi_{j}\right)\right] .
$$

where $\Delta \phi_{i}$ are defined as the azimuthal angles corresponding to the angles $\theta_{i}$ above. At LO we found $\mathscr{D}^{\prime}=-0.193$.

\section{Parton and hadron level correlations}

For a $2 \mathrm{HDM}$ (II) with a charged Higgs mass below the top mass the main decay channel is to $\tau v_{\tau}$. We have examined the correlations at parton level and hadron level for top pair production in 

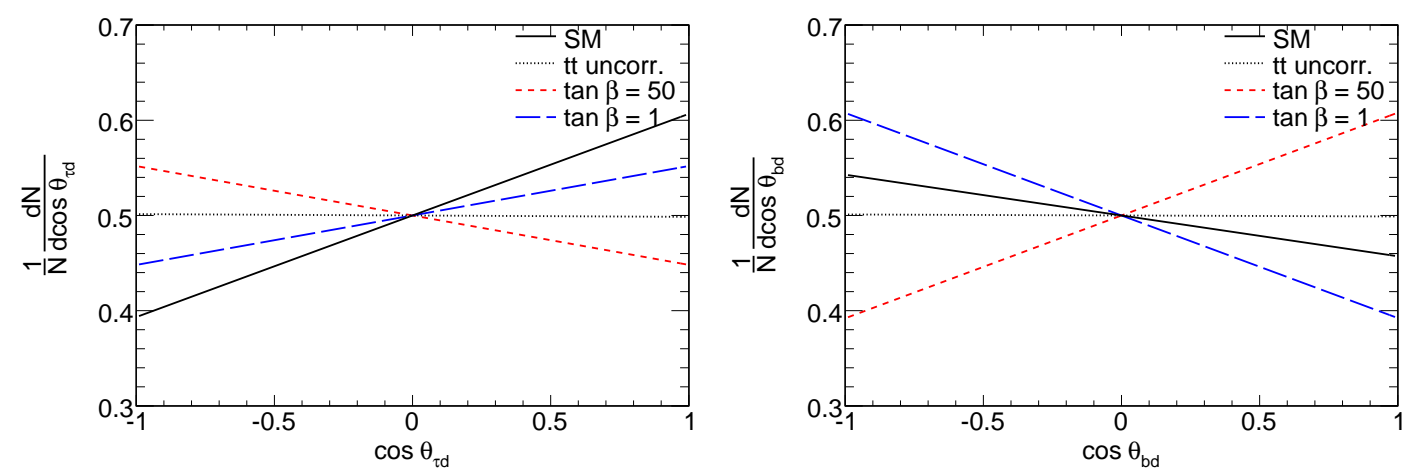

Figure 2: Parton level correlation of $\mathscr{D}$-type for the pairs $t d$, left, and $b d$, right. In black solid line is the full SM and the black dotted line is SM without any correlations. The 2HDM(II) is shown in red, short dashed line, for $\tan \beta=50$ and in blue, long dashed line, for $\tan \beta=1$.

SM and 2HDM(II) with a charged Higgs mass of around $80 \mathrm{GeV}$. For the decays of the top quarks we have assumed $t \rightarrow b H^{+} / W^{+} \rightarrow b \tau^{+} v_{\tau}$ and $\bar{t} \rightarrow \bar{b} W^{-} \rightarrow \bar{b} \bar{u} d$. As seen in table 2 the best analyzing particles to use for decays via $W$ are leptons or $d$ quarks whereas for $H^{+}$in 2HDM(II) the $b$ quark or $H^{+}$itself is better. In figure 2 the correlations are shown for uncorrelated top pairs and correlated top pairs in SM and 2HDM(II) with $\tan \beta=50$ and $\tan \beta=1$.

The $\mathscr{D}$ type correlations given here are not measurable since they require that the complete event is reconstructed, including the four vector for the $\tau$ and $v_{\tau}$, which is not possible experimentally since the $\tau$ decays further. As said above azimuthal correlations can be used without full reconstruction. To be more realistic we do not look at the $d$ quark, since it is hard to experimentally distinguish a $d$ jet form a $u$ jet, but instead use the $b$ on the $W$ side. The two upper plots in figure 3 shows parton level $\mathscr{D}^{\prime}$ correlations. The correlations here are small but should be measurable. We have therefore also done a hadron level study of these correlations. We used MadGraph/MadEvent 4.1.10 [4] to simulate the full $2 \rightarrow 6$ processes with subsequent hadronic decay of the $\tau$ with Tauola [5] and parton showering and hadronization with Pythia 6.409 [6]. A simple analysis is then preformed where we start by doing a $k_{\perp}$ jet finding in $|\eta|<5$ with $d_{c u t}=20 \mathrm{GeV}$. "Flavor tagging" was done by requiring $\Delta R($ jet, truth $)<0.4$ in $|\eta|<2.5$. $W^{+}$candidates were defined as $\left|m_{j j}-m_{W^{+}}\right|<10$ $\mathrm{GeV}$ and $t$ candidate as $\left|m_{j j b}-m_{t}\right|<15 \mathrm{GeV}$. No detector effects where included and the analysis was done for the different samples individually. The result at hadron level shown in the two lower plots of figure 3 correspond to roughly $10 \mathrm{fb}^{-1}$. The main difference between parton level and hadron level is the limited statistics, the correlations are still visible and the uncorrelated sample is still uncorrelated.

\section{Conclusions}

Top quarks produced at hadron colliders have their spin correlated. The correlation is seen in the angular distributions of the decay products. When the top decay can be mediated by a charged Higgs the angular correlations of the decay products differ from the pure SM case. Since the charged Higgs dominantly decays to tau leptons one has to resort to azimuthal correlations. We 

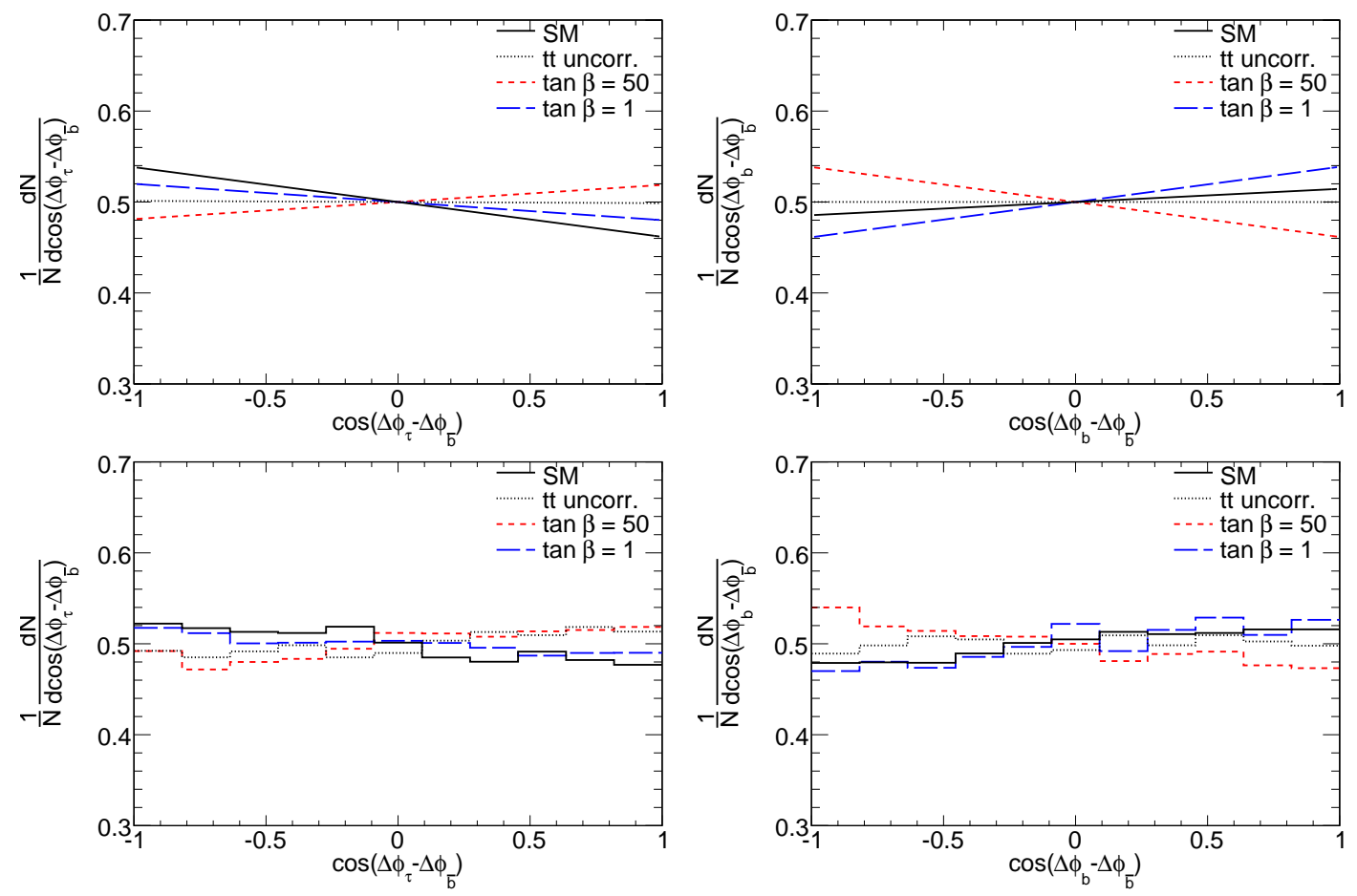

Figure 3: $\mathscr{D}^{\prime}$-type for the pairs $\tau b$, left, and $b b$, right. The upper plots are at parton level and the lower at hadron level. In black solid line is the full SM and the black dotted line is SM without any correlations. The $2 \mathrm{HDM}(\mathrm{II})$ is shown in red, short dashed line, for $\tan \beta=50$ and in blue, long dashed line, for $\tan \beta=1$.

have showed that, although small, measurable azimuthal correlations exist and that they survive at hadron level.

\section{Acknowledgments}

I would like to that my collaborators Gunnar Ingelman, Johan Rathsman and Oscar Stål.

\section{References}

[1] D. Eriksson, G. Ingelman, J. Rathsman, and O. Stal, New angles on top quark decay to a charged Higgs, JHEP 01 (2008) 024, [arXiv: 0710.5906 ].

[2] T. Stelzer and S. Willenbrock, Spin correlation in top-quark production at hadron colliders, Phys. Lett. B 374 (1996) 169-172.

[3] W. Bernreuther, A. Brandenburg, Z. G. Si, and P. Uwer, Top quark pair production and decay at hadron colliders, Nucl. Phys. B690 (2004) 81-137, [hep-ph/ 0403035$].$

[4] J. Alwall et. al., MadGraph/MadEvent v4: The New Web Generation, arXiv:0706.2334 [hep-ph].

[5] S. Jadach, Z. Was, R. Decker, and J. H. Kuhn, The tau decay library TAUOLA: Version 2.4, Comput. Phys. Commun. 76 (1993) 361-380.

[6] T. Sjöstrand, S. Mrenna, and P. Skands, PYTHIA 6.4 physics and manual, JHEP 05 (2006) 026, [hep-ph/0603175]. 